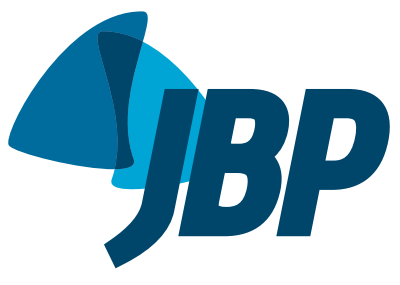

\title{
Validation of the Pulmonary Embolism Severity Index for risk stratification after acute pulmonary embolism in a cohort of patients in Brazil
}

\author{
Leonardo de Araujo Soriano, ,a, Talita Tavares Castro, b, Kelvin Vilalva',c, \\ Marcos de Carvalho Borges ${ }^{1, \mathrm{~d}}$, Antonio Pazin-Filho ${ }^{1, \mathrm{e}}$, Carlos Henrique Miranda ${ }^{1, f}$
}

1. Divisão de Emergências Clínicas, Departamento de Clínica Médica Faculdade de Medicina de Ribeirão Preto, Universidade de São Paulo, Ribeirão Preto (SP) Brasil.

a. (D) http://orcid.org/0000-0003-2679-9642

b. (D) http://orcid.org/0000-0001-5994-9278

c. (D) http://orcid.org/0000-0003-4407-2906

d. (D) http://orcid.org/0000-0001-6280-0714

e. (iD) http://orcid.org/0000-0001-5242-329X

f. (D) http://orcid.org/0000-0002-5968-4879

Submitted: 24 July 2017.

Accepted: 12 August 2018

Study carried out in the Divisão de Emergências Clínicas, Departamento de Clínica Médica, Faculdade de Medicina de Ribeirão Preto, Universidade de São Paulo, Ribeirão Preto (SP) Brasil.

\begin{abstract}
Objective: To validate the Pulmonary Embolism Severity Index (PESI), which was developed for risk stratification after acute pulmonary embolism (PE), for use in Brazil. Methods: This was a single-center retrospective study involving patients admitted to the emergency department with acute PE. The original and simplified versions of the PESI were calculated using hospital admission data from medical records. The outcome measure was the overall 30-day mortality rate. Results: We included 123 patients. The mean age was $57 \pm 17$ years, and there was a predominance of females, who accounted for $60 \%$ of the cohort. There were 28 deaths, translating to an overall 30 -day mortality rate of $23 \%$. In the cluster analysis by risk class, overall 30 -day mortality was $2.40 \%$ for classes I-II, compared with $20.00 \%$ for classes III-IV-V (relative risk [RR] = 5.9; 95\% Cl: 1.88-18.51; $p=0.0002$ ). When we calculated overall 30-day mortality using the simplified version (0 points vs. $\geq 1$ point), we found it to be $3.25 \%$ for 0 points and $19.51 \%$ for $\geq 1$ point $(R R=2.38 ; 95 \% \mathrm{Cl}$ : $0.89-6.38 ; p=0.06)$. Using the original version, a survival analysis showed that risk classes I and II presented similar KaplanMeier curves ( $p=0.59$ ), as did risk classes III, IV, and V $(p=0.25)$. However, the curve of the clusters based on the original version, showed significantly higher mortality in the III-IV-V classes than in the I-II classes (RR = 7.63; 95\% CI: 2.29-25.21; $p=0.0001$ ). The cluster analysis based on the original version showed a greater area under the ROC curve than did the analysis based on the simplified version $10.70 ; 95 \% \mathrm{Cl}: 0.62-0.77$ vs. 0.60; $95 \% \mathrm{Cl}: 0.51-0.67 ; p=0.05)$. Conclusions: The PESI adequately predicted the prognosis after acute PE in this sample of the population of Brazil. The cluster analysis based on the original version is the most appropriate analysis in this setting.
\end{abstract}

Keywords: Pulmonary embolism; Severity of illness index; Risk assessment.

\section{INTRODUCTION}

Acute pulmonary embolism (PE) is a prevalent disease with a broad spectrum of clinical presentation, ranging from asymptomatic (an incidental finding on CT) to severe hemodynamic instability and sudden death. ${ }^{(1)}$ In this diverse scenario, some tools have been proposed to help stratify the risk of unfavorable outcomes over the clinical course of acute PE.

The Pulmonary Embolism Severity Index (PESI) is a tool that was developed from a retrospective study of data from a large database of records for patients treated in the United States; the study included an initial sample of 10,354 patients who were discharged from the hospital with a diagnosis of acute PE and a subsequent subsample of 5,177 patients for internal validation. (2) The objective of the PESI was to stratify the risk of death after identification of acute $\mathrm{PE}$, on the basis of objective clinical parameters, in order to help guide treatment. A logistic regression model identified 11 clinical variables as independent predictors of overall 30-day mortality. A model with $\beta$ coefficients attributed a different weight to each of those variables. On the basis of the total score on the PESI, ${ }^{(2)}$ patients were grouped into five categories (Table 1): risk class I (very low risk); risk class II (low risk); risk class III (intermediate risk); risk class IV (high risk); and risk class $\mathrm{V}$ (very high risk).

The PESI has been validated in population-based samples from different countries. ${ }^{(3-5)}$ In 2014, the European guidelines for the diagnosis and treatment of acute PE included this tool in their treatment guidance flowchart. ${ }^{(1)}$ However, to our knowledge, there have been no studies validating the PESI for use in Brazil. The objective of the present study was to validate the PESI in a retrospective cohort of patients with a diagnosis of acute PE in Brazil.

\section{METHODS}

This was a single-center retrospective cohort study involving patients admitted to the Emergency Department of the University of São Paulo at Ribeirão Preto School

Correspondence to:

Carlos Henrique Miranda. Centro Integrado de Emergências em Saúde, Unidade de Emergência - HCFMRP-USP - Rua Bernardino de Campos, 1000 , CEP

14020-670, Ribeirão Preto, SP, Brasil.

Tel.: 55 16 3602-1240. Fax: 5516 3602-1240. E-mail: chmiranda@fmrp.usp.br

Financial support: None. 
Table 1. Parameters used in the Pulmonary Embolism Severity Index for overall 30-day mortality risk stratification after acute pulmonary embolism.

\begin{tabular}{lcc}
\multicolumn{1}{c}{ Parameter } & Original version & Simplified version \\
Age, years & $+n$ of years & 1 point (if $>80$ years) \\
Male gender & +10 points & - \\
Cancer & +30 points & 1 point \\
Heart failure & +10 points & 1 point \\
COPD & +10 points & 1 point \\
$\mathrm{HR} \geq 110 \mathrm{bpm}$ & +20 points & 1 point \\
$\mathrm{SBP}<100 \mathrm{mmHg}$ & +30 points & - \\
$\mathrm{RR}>30$ breaths $/ \mathrm{min}$ & +20 points & - \\
Temperature $<36^{\circ} \mathrm{C}$ & +20 points & - \\
Altered mental status & +60 points & 1 point \\
SpO $<90 \%$ & +20 points & \\
Risk stratification (based on the total score) & & 0 points \\
Class I & $<65$ points & $\geq 1$ point \\
Class II & $65-85$ points & \\
Class III & $86-105$ points & \\
Class IV & $106-125$ points & \\
Class V & $>125$ points & \\
\hline
\end{tabular}

SBP: systolic blood pressure.

of Medicine Hospital das Clínicas, located in the city of Ribeirão Preto, Brazil, with a primary diagnosis of acute PE. The hospital, which is dedicated to emergency care, is a tertiary referral center for the 26 municipalities within the XIII Regional Health Care Division of the São Paulo State Health Department and treats an average of approximately 20,000 patients per year. The study was approved by the local research ethics committee (Ruling no. 919/2016) and was conducted in accordance with the Declaration of Helsinki.

\section{Data collection}

We reviewed the medical records of all patients admitted to the emergency department between January of 2009 and December of 2015 with a primary diagnosis of acute $\mathrm{PE}$, recorded on electronic discharge forms as code I26.0 (pulmonary embolism with acute cor pulmonale) or I26.9 (pulmonary embolism without acute cor pulmonale) of the International Classification of Diseases, 10th revision (ICD-10). A definitive diagnosis of acute PE was defined as the presence of a clinical profile consistent with acute PE plus at least one confirmatory criterion: filling defects on CT angiography of the pulmonary arteries; ventilation/perfusion lung scintigraphy findings of perfusion defects in ventilated areas (high probability); intraluminal filling defects on conventional pulmonary angiography; leg ultrasound findings consistent with deep vein thrombosis; or autopsy findings identifying lobar or central embolism without evidence of any other alternative diagnoses.

For those patients with a definitive diagnosis of acute $P E$, we calculated the original and simplified versions of the PESI. To that end, we used clinical data from medical records created at hospital admission. If any parameter used in the PESI was missing from the medical record, the index was calculated in the same way, without the inclusion of that missing item. According to the values obtained, patients were classified into one of the five risk classes of the original version and into one of the two risk classes of the simplified version, as described in the literature (Table 1 ). Other demographic and clinical data that are not used in the PESI were obtained through a review of medical records.

The outcome measure assessed in the present investigation was the overall 30-day mortality rate, an outcome measure that is identical to that of the original study. (2) Patients who were discharged from the hospital before completing the 30-day follow-up were contacted by telephone. The telephone contact was made by a member of the clinical research unit of our institution, who was properly trained in analyzing survival. When a death was confirmed, the date of death was requested.

\section{Statistical analysis}

Categorical variables were expressed as frequency and proportion. Continuous variables with normal distribution were expressed as mean and standard deviation, whereas the other variables were expressed as median and interquartile range. To compare overall 30-day mortality rates across the different risk classes, we calculated relative risk (RR) and the corresponding $95 \% \mathrm{CI}$, subsequently applying the chi-square test. To analyze survival, we constructed various Kaplan-Meier curves, which we compared using the log-rank test. To compare prognostic accuracy between the original version and the simplified version, we constructed ROC curves and examined the area under the curve (AUC) for each. Given the study design, no sample size estimation was performed. Statistical significance was defined as a two-tailed $p \leq 0.05$. We performed the statistical analysis and constructed graphs using 
STATA software, version 13.1 (StataCorp LP, College Station, TX, USA).

\section{RESULTS}

During the study period, 231 patients with a primary diagnosis of acute PE were admitted to the emergency department. After a detailed review of medical records, 123 patients were found to meet the criteria for a definitive diagnosis of acute PE according to the parameters established in the present study.

For 6 of the 11 parameters used to calculate the PESI, data were available in the medical records of all 123 patients included in the study. The proportions of patients for whom medical record data were available for the other 5 parameters were as follows: $99 \%$ for altered mental status; $97 \%$ for systolic blood pressure < $100 \mathrm{mmHg}$; $93 \%$ for RR > 30 breaths/min; 89\% for temperature $<36^{\circ} \mathrm{C}$; and $89 \%$ for $\mathrm{SpO}_{2}<90 \%$.

The demographic, clinical, and laboratory characteristics of these patients with a definitive diagnosis of acute PE are shown in Table 2. The mean age of the patients was $57 \pm 17$ years, and there was a predominance of females, who accounted for $60 \%$ of the cohort. The most common clinical finding was dyspnea (in $81 \%$ ), followed by any chest pain (in 33\%), leg pain (in 33\%), cough (in 32\%), signs of deep vein thrombosis (in 30\%), and pleuritic chest pain (in 29\%). The other signs and symptoms were less prevalent. The median time to the onset of the clinical presentation was 3 days (range: 1-18 days). The presence of circulatory shock and cardiopulmonary arrest was observed in 13 patients (11\%) and 9 patients (7\%), respectively. The most common predisposing factors were obesity (in 38\%), immobilization for more than 3 days (in $31 \%$ ), previous deep vein thrombosis (in $23 \%$ ), and recent surgery (in 15\%). The presence of active neoplasia was documented in 8 patients (7\%).

CT angiography was the diagnostic imaging test most commonly used (in $80 \%$ ); ventilation/perfusion lung scintigraphy and ultrasound of the lower limbs were used in smaller proportions of patients. Conventional pulmonary angiography was not used as a diagnostic instrument in any of the cases analyzed. The definitive diagnosis was confirmed by autopsy in only 5 patients, all of whom were admitted with cardiopulmonary arrest and rapidly progressed to death before any confirmatory imaging test was performed.

With regard to treatment, 32 patients (26\%) received thrombolytic agents; of those, 22 were hemodynamically unstable and 10 were hemodynamically stable. The use of thrombolytic agents in this latter group remains controversial in the literature, and these cases were selected by the medical team for this type of treatment because of significant functional impairment, the need for increased supplemental oxygen, high thrombotic load, marked pulmonary hypertension, and right ventricular dysfunction. Most of the patients received systemic heparinization, with low-molecular-weight heparin (in $73 \%$ ) or unfractionated heparin (in 20\%).
Table 2. Demographic, clinical, and laboratory characteristics of the patients diagnosed with acute pulmonary embolism who were included in the study, at the time of hospital admission $(\mathrm{N}=123)$. ${ }^{\text {a }}$

\begin{tabular}{|c|c|}
\hline Characteristic & Result \\
\hline \multicolumn{2}{|l|}{ Demographic } \\
\hline Age, years & $57 \pm 18$ \\
\hline Male gender & $49(40)$ \\
\hline \multicolumn{2}{|l|}{ Race } \\
\hline White & $100(81)$ \\
\hline Black & $23(19)$ \\
\hline \multicolumn{2}{|l|}{ Clinical findings } \\
\hline Dyspnea & $100(81)$ \\
\hline Any chest pain & $40(33)$ \\
\hline Leg pain & $40(33)$ \\
\hline Cough & $39(32)$ \\
\hline Signs of DVT & $37(30)$ \\
\hline Pleuritic chest pain & $36(29)$ \\
\hline Syncope & $20(16)$ \\
\hline Fever & $17(14)$ \\
\hline Circulatory shock & $13(11)$ \\
\hline Hemoptysis & $11(09)$ \\
\hline Cardiopulmonary arrest & $09(07)$ \\
\hline Duration of symptoms, days & $03[1-6]$ \\
\hline $\mathrm{RR}$, breaths/min & $24[19-30]$ \\
\hline $\mathrm{SpO}_{2}$ on room air, $\%$ & 92 [87-95] \\
\hline $\mathrm{SBP}, \mathrm{mmHg}$ & 120 [110-130] \\
\hline $\mathrm{DBP}, \mathrm{mmHg}$ & $75[70-90]$ \\
\hline $\mathrm{HR}$, bpm & 96 [84-109] \\
\hline Shock index (HR/SBP) & $0.87 \pm 0.27$ \\
\hline \multicolumn{2}{|l|}{ Risk factors } \\
\hline Obesity & $47(38)$ \\
\hline Immobilization $>3$ days & $38(31)$ \\
\hline Previous DVT & $28(23)$ \\
\hline Recent surgery $<1$ month prior & $18(15)$ \\
\hline Fracture & $17(14)$ \\
\hline Heart failure & $16(13)$ \\
\hline Previous stroke & $15(12)$ \\
\hline Oral contraceptive use & $13(11)$ \\
\hline Thrombophilia & $12(10)$ \\
\hline COPD & $10(08)$ \\
\hline Active neoplasia & $08(07)$ \\
\hline \multicolumn{2}{|l|}{ Diagnostic method } \\
\hline CT angiography & $99(80)$ \\
\hline DVT ultrasound of legs & $11(09)$ \\
\hline V/P lung scintigraphy & $08(07)$ \\
\hline Autopsy & $05(04)$ \\
\hline \multicolumn{2}{|l|}{ Treatment } \\
\hline Low-molecular-weight heparin & $90(73)$ \\
\hline Thrombolytic agents & $32(26)$ \\
\hline Unfractionated heparin & $24(20)$ \\
\hline Vena cava filter & $03(02)$ \\
\hline
\end{tabular}

SBP: systolic blood pressure; DBP: diastolic blood pressure; DVT: deep vein thrombosis; and V/P: ventilation/perfusion. ${ }^{a}$ Values expressed as mean \pm $\mathrm{SD}, \mathrm{n}(\%)$, or median [interquartile range]. 
Nine patients did not receive any type of heparinization, 5 because of rapid progression to death, due to the fact that they were admitted with cardiopulmonary arrest, and 4 because of contraindications, such as coagulopathy, thrombocytopenia, or bleeding. Of the 4 patients with contraindications, 1 underwent surgical embolectomy and 3 received an inferior vena cava filter.

There were 28 deaths, translating to an overall 30 -day mortality rate of $23 \%$. Among the patients who died, the PESI risk classes were distributed as follows: class I, in $0.80 \%$; class II, in $1.70 \%$; class III, in $4.90 \%$; class IV, in 3.25\%; and class V, in $12.20 \%$ ( $p<0.0001)$. The cluster analysis based on the original version showed that overall 30-day mortality was higher for classes III-IV-V (20.00\%) than for classes I-II $(2.40 \%)-\mathrm{RR}=5.9 ; 95 \% \mathrm{CI}$ : $1.88-18.51 ; p=0.0002 ;$ with a negative predictive value of $94 \%$ and a positive predictive value of $35 \%$. When we calculated overall 30-day mortality using the simplified PESI (0 points vs. $\geq 1$ point), we found it to be $3.2 \%$ for 0 points and $19.5 \%$ for $\geq 1$ point ( $R R=$ 2.38 ; 95\% CI: 0.89-6.38; $\mathrm{p}=0.06$ ), with a negative predictive value of $88 \%$ and a positive predictive value of 35\% (Table 3).

A survival analysis with Kaplan-Meier curves, using the original version, showed that risk classes I and II presented similar curves ( $p=0.59)$, as did risk classes III, IV, and V ( $p=0.25)$. However, a comparison of the Kaplan-Meier curves of the clusters based on the original version showed significantly higher overall 30 -day mortality in risk classes III-IV-V than in risk classes I-II (RR = 7.63; 95\% CI: 2.29-25.21; p = 0.0001 ; Figure 1). An analysis of the Kaplan-Meier curves for the simplified version ( 0 points vs. $\geq 1$ point) showed that overall 30-day mortality was higher for 0 points than for $\geq 1$ point $(R R=2.95 ; 95 \% C I$ : 1.02-8.51; $\mathrm{p}=0.03$ ).

An ROC curve comparison of prognostic accuracy for determining overall 30-day mortality revealed that the cluster analysis based on the original version had greater accuracy than did the analysis based on the simplified version, with an AUC of 0.70 (95\% CI: $0.62-0.77)$ vs. 0.60 (95\% CI: $0.51-0.67 ; p=0.05$; Figure 2).

\section{DISCUSSION}

The present investigation showed that the PESI adequately predicted the prognosis after an episode of acute PE in this sample of the population of Brazil and that the cluster analysis based on the original version is the most appropriate way to use this tool.

One notable finding of the present study is the high overall 30 -day mortality rate (23\%). In addition, the prevalence of circulatory shock in the initial clinical presentation was high (11\%). A study conducted in the United States, ${ }^{(6)}$ using real-world records of patients diagnosed with acute PE in the emergency department, reported an overall 30 -day mortality rate of $5.4 \%$ and a prevalence of circulatory shock of $3.0 \%$ in a populationbased sample with demographic characteristics very similar to those observed in our investigation, both in terms of age $(56 \pm 18$ years vs. $57 \pm 18$ years; $p$ $=0.766)$ and in terms of a predominance of females (53\% vs. $60 \% ; p=0.11$ ). ${ }^{(6)}$ We speculate that several factors, such as delayed diagnosis of acute PE and delayed initiation of heparinization in patients in a public hospital setting in Brazil, could be responsible for these findings in our study.

To our knowledge, there is only one multicenter registry in Brazil that included 727 patients with a diagnosis of acute PE admitted to the emergency department or the ICU and that also found a high in-hospital mortality rate $(19.5 \%),{ }^{(7)}$ which was very similar to that reported in our investigation. A separate analysis of data from that same registry also showed a high (14.1\%) overall mortality rate even in the subgroup of hemodynamically stable patients. ${ }^{(8)}$

Table 3. Data on prevalence and overall 30-day mortality observed in patients with acute pulmonary embolism in the present study, by classification according to the original Pulmonary Embolism Severity Index (PESI), the clusters based on the original PESI, and the simplified PESI, and comparison with data reported in the international literature. ${ }^{\mathrm{a}}$ Classification Prevalence Overall 30-day mortality Variation in overall 30-day

Original version

Risk class I, very low

25 (20)

$26(21)$

$24(20)$

Risk class III, moderate

$15(12)$

$33(27)$

$123(100)$

Risk class IV, high

$123(100)$

Total

$72(59)$
$01(0.80)$

02 (1.70)

$06(4.90)$

04 (3.25)

$15(12.20)$

$28(23.00)$ mortality $(\%)^{\mathrm{b}}$

\section{Clusters based on the original version}

0.0-1.6

1.7-3.5

3.2-7.1

4.0-11.4

$10.0-24.5$

\begin{tabular}{llcl} 
Risk classes I-II & $51(41)$ & $03(02.40)$ & \\
Risk classes III-IV-V & $72(59)$ & $25(20.00)$ & $\begin{array}{l}\text { Variation in overall 30-day } \\
\text { mortality, \% (95\% CI) }\end{array}$ \\
Simplified version & & & $1.0(0.0-2.1)$ \\
& & $04(03.20)$ & $10.9(8.5-13.2)$ \\
\hline 1 points & $35(28)$ & $24(19.50)$ & $88(72)$ \\
\hline
\end{tabular}

aalues expressed as $\mathrm{n}(\%)$, except where otherwise indicated. ${ }^{\mathrm{b}}$ According to references $1-5$. 
(A)

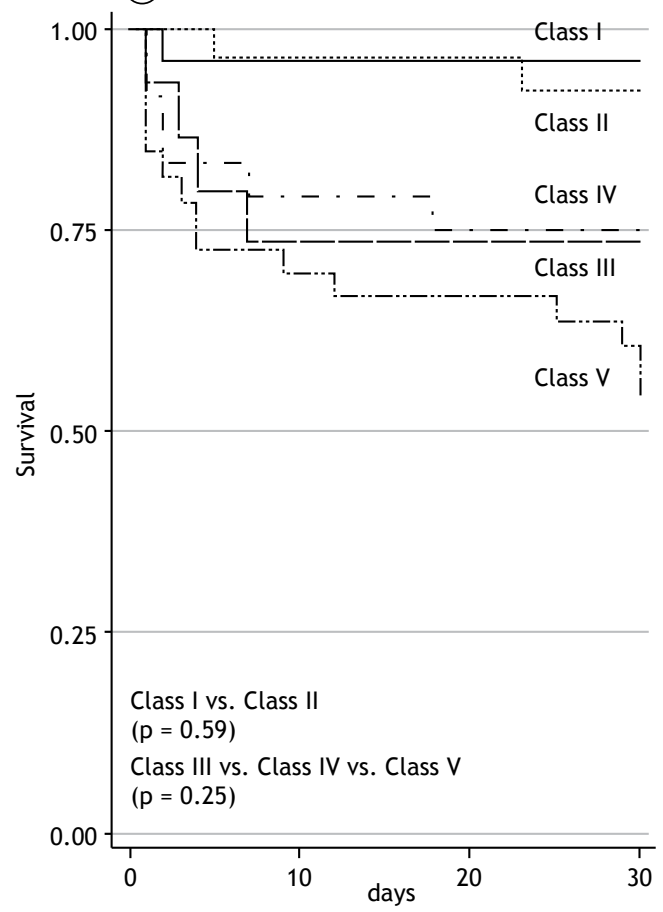

(B)

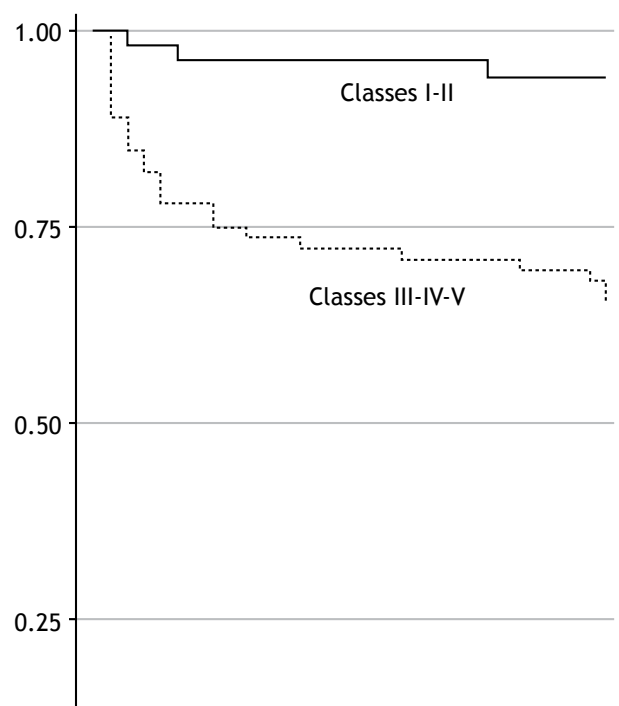

Classes I-II vs. Classes III-IV-V

RR: $7.63 ; 95 \% \mathrm{Cl}: 2.29-25.21 ; \mathrm{p}=0.0001$

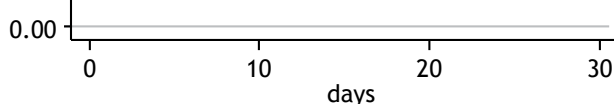

Figure 1. Kaplan-Meier curves showing 30-day survival in patients diagnosed with pulmonary embolism, by each of the five Pulmonary Embolism Severity Index risk classes (in A) and by cluster (risk classes I-II vs. risk classes III-IV-V; in B). RR: relative risk.

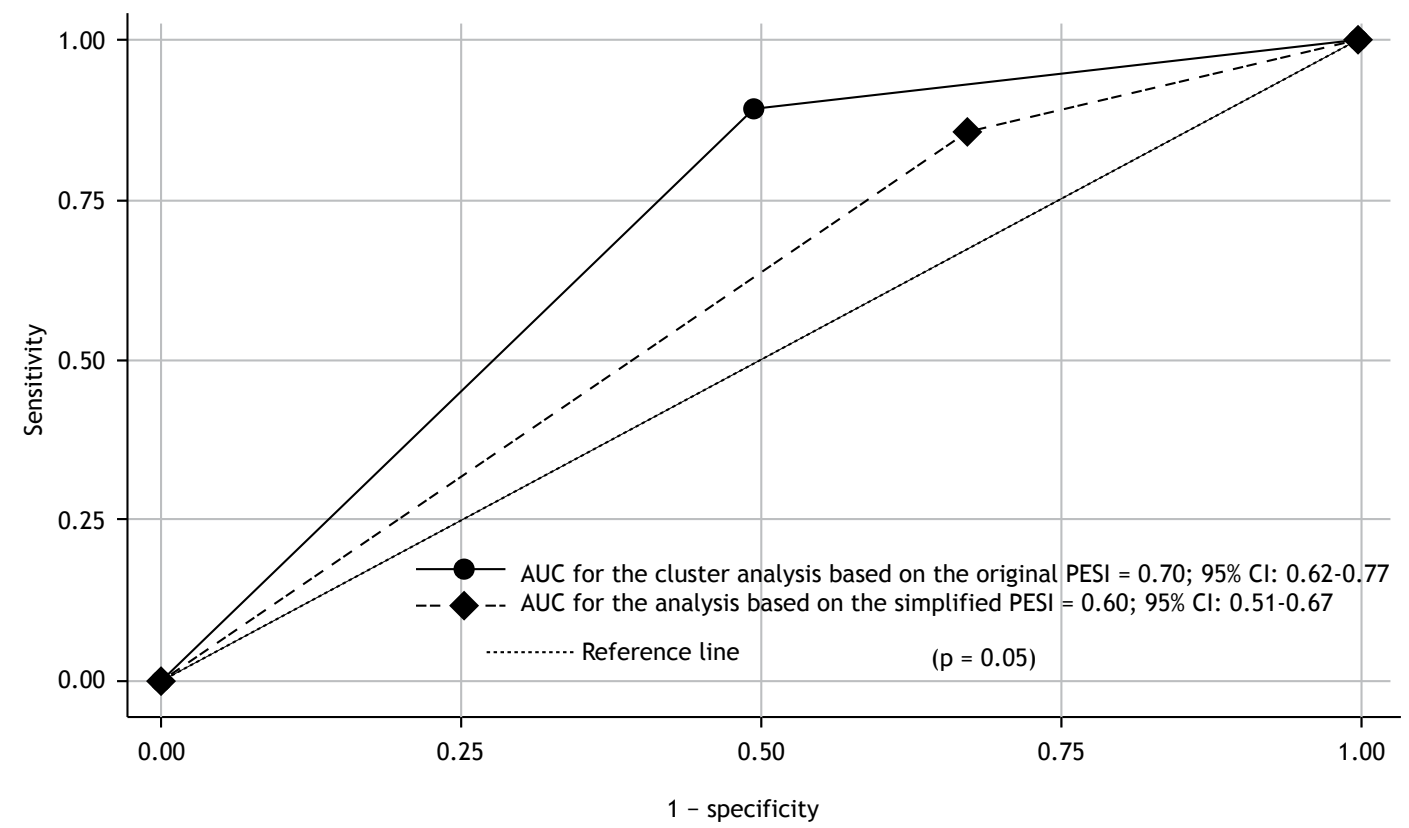

Figure 2. ROC curve comparison of prognostic accuracy for predicting overall 30-day mortality after acute pulmonary embolism between the cluster analysis based on the original Pulmonary Embolism Severity Index (PESI) and the analysis based on the simplified PESI. AUC: area under the (ROC) curve.

A large international registry ${ }^{(9)}$ showed an overall 30 -day mortality rate of 3.3\% (95\% CI: $2.9-3.8 \%)$, which is also very similar to that reported by Pollack et al. ${ }^{(6)}$ In contrast, a study by Goldhaber et al. ${ }^{(10)}$ showed a 14-day mortality rate of $11.2 \%$ (95\% CI: $10.0-12.5 \%$ ), which is higher than that found in other international registries, ${ }^{(8,9)}$ probably because it included an older population. It is important to emphasize that the data of the present investigation are mainly related to a primary diagnosis of acute PE as the cause of hospital admission and that these data are not representative of acute nosocomial PE. 
When we analyzed overall 30-day mortality by PESI risk class, we found that the rates were similar to those reported in the international literature, ${ }^{(1-5)}$ especially those reported in the study that originally described the PESI. (2) The exception was risk class IV, for which the overall 30-day mortality rate was slightly lower in our study than in the first validation study $(3.25 \%$ vs. $4.0-11.0 \%)$. $^{(2)}$ It is worth noting that risk class IV had the lowest prevalence in our study (12.20\%), as it did in the original study, ${ }^{(2)}$ where its prevalence ranged from $11.30 \%$ to $16.40 \%$. Another important factor is that, in the study that described the PESI, ${ }^{(2)}$ the overall 30-day mortality rate for risk class IV was higher in the initial sample and the internal validation sample $(10.40 \%$ and $11.40 \%$, respectively), whereas it was proportionally lower $(4.0 \%)$ in the external PESI validation sample. ${ }^{(2)}$ This last value is very close to that observed in our study. In addition, as has been shown in the scientific literature, ${ }^{(11)}$ our investigation showed that the cluster analysis based on the original version, in which risk classes III-IV-V are analyzed as a group rather than individually, seems to be the most appropriate. ${ }^{(11)}$

One of the major advantages related to the use of the PESI is the selection of a group of patients at low risk for complications, characterized as risk class I-II, who may be discharged from the hospital early and undergo home treatment, thus reducing hospital costs. ${ }^{(12-14)}$ Our investigation also showed that the prognosis was good in the patients in this group (risk class I-II), with a reduced overall 30-day mortality rate. Nevertheless, it is worth noting that early hospital discharge resulting from the use of new anticoagulants does not typically occur at the locale under study, especially during the period evaluated.

Like other prognostic markers in the assessment of acute $P E$, the cluster analysis based on the original PESI has a high negative predictive value $(94 \%)$ but a low positive predictive value (35\%). Therefore, the PESI is more useful in selecting those patients with a good prognosis, and the presence of patients in risk classes III-IV-V does not necessarily imply the occurrence of adverse events and the need for more aggressive therapy. ${ }^{(15)}$ The 2014 European guidelines for the management of acute $\mathrm{PE}^{(1)}$ recommend the use of a new risk stratification method based on biomarkers such as troponin and NT-proBNP, as well as on imaging tests for assessing the right ventricle, in patients in risk classes III-IV-V.

Our results are also in line with those in the literature ${ }^{(11)}$ : the cluster analysis based on the original version (risk classes I-II vs. risk classes III-IV-V) seems to be the most appropriate way to use this instrument, as shown by the good evidence obtained from the Kaplan-Meier curves (Figure 1). This form of analysis divides patients into two groups with very different prognosis (overall 30-day mortality of $2.40 \%$ vs. overall 30 -day mortality of $20.00 \%$ ). A recent systematic review and meta-analysis ${ }^{(11)}$ that evaluated prognostic models in acute PE showed overall 30 -day mortality rates of $2.30 \%$ and $11.40 \%$, respectively, in the low-risk group (PESI risk classes I-II) and high-risk group (PESI risk classes III-IV-V). The mortality observed in the low-risk group in our investigation $(2.40 \%)$ is similar to the $2.30 \%$ observed in that study. ${ }^{(11)}$

Given that numerous variables are involved in the original PESI, an attempt was made to develop a simplified version including only 6 variables that have similar weights in the calculation of the index. That version is known as simplified PESI. One early study showed no difference in prognostic accuracy between the original version and the simplified version, with identical AUC of 0.75 (95\% CI: 0.69-0.80; $p=0.95)$. ${ }^{(16)}$ However, a subsequent investigation showed that the prognostic accuracy of the original version was higher than that of the simplified version, with an AUC of 0.78 (95\% CI: $0.77-0.79)$ vs. 0.72 (95\% CI: $0.71-0.74 ; \mathrm{p}<0.001)$, ${ }^{(17)}$ similarly to our investigation, in which the original version was also found to be slightly superior to the simplified version, with an AUC of 0.70 (95\% CI: $0.62-0.77)$ vs. 0.60 (95\% CI: $0.51-0.67 ; p=0.05)$. It is important to emphasize that, in our investigation, the prognostic accuracies of both versions were slightly lower than those reported in the international literature. ${ }^{(17)}$

Some limitations of the present investigation are worthy of note. First, because data were collected retrospectively from medical records, not all of the data needed to calculate the PESI were available for some patients. That may have resulted in the final value of the PESI being underestimated in a small portion of the sample. However, the proportion of individuals with incomplete medical records was small and therefore had little influence on the final results of the present investigation. In addition, it was possible to assess the outcome measure (overall 30-day mortality) in all of the patients included in the present analysis. It is worth emphasizing that the outcome assessed was overall 30-day mortality, which does not necessarily reflect mortality associated with acute PE; however, most studies validating the PESI have also used this same outcome. ${ }^{(2,4)}$ Second, this was a single-center study conducted at a tertiary referral hospital dedicated to emergency care, which may have led to the selection of patients with greater disease severity, potentially creating a selection bias, similarly to that seen in another study on acute PE conducted in Brazil. ${ }^{(7)}$ Third, our retrospective review of patient medical records was based on the ICD-10 codes recorded on electronic discharge forms. That may have caused the non-inclusion of some patients. Fourth, because the PESI is based on quantitative clinical parameters that are quite objective, we considered that other steps of the validation process, such as back-translation and cross-cultural validation, were unnecessary. A positive point of our investigation was that confirmation of the diagnosis by complementary imaging was mandatory, unlike many of the studies validating the PESI that have been published in the literature, ${ }^{(2,4)}$ all of which 
used only hospital admission databases coded for acute $\mathrm{PE}$ and did not require confirmation of the diagnosis by imaging.

The PESI adequately predicted the prognosis after acute PE in this sample of the population of Brazil. The cluster analysis based on the original version is the most appropriate way to use this tool in this setting. Although the overall 30-day mortality rate after acute PE observed in our sample is high when compared with that reported in international studies, $(6,9,10)$ it is in agreement with data from a study on this topic conducted in Brazil. ${ }^{(7)}$ It is necessary that prospective multicenter studies be conducted in Brazil, in order to further assess mortality associated with acute PE in our population.

\section{REFERENCES}

1. Konstantinides SV, Torbicki A, Agnelli G, Danchin N, Fitzmaurice D, Galie N, et al. 2014 ESC guidelines on the diagnosis and management of acute pulmonary embolism. Eur Heart J. 2014;35(43):3033-69, 3069a-3069k

2. Aujesky D, Obrosky DS, Stone RA, Auble TE, Perrier A, Cornuz J, et al. Derivation and validation of a prognostic model for pulmonary embolism. Am J Respir Crit Care Med. 2005;172(8):1041-6. https:// doi.org/10.1164/rccm.200506-8620C

3. Chan CM, Woods C, Shorr AF. The validation and reproducibility of the pulmonary embolism severity index. J Thromb Haemost. 2010;8(7):1509-14. https://doi.org/10.1111/j.15387836.2010.03888.x

4. Donzé J, Le Gal G, Fine MJ, Roy PM, Sanchez O, Verschuren F, et al. Prospective validation of the Pulmonary Embolism Severity Index. A clinical prognostic model for pulmonary embolism. Thromb Haemost. 2008;100(5):943-8. https://doi.org/10.1160/TH08-05-0285

5. Chan CM, Woods CJ, Shorr AF. Comparing the pulmonary embolism severity index and the prognosis in pulmonary embolism scores as risk stratification tools. J Hosp Med. 2012;7(1):22-7. https://doi. org/10.1002/jhm.932

6. Pollack CV, Schreiber D, Goldhaber SZ, Slattery D, Fanikos J, O'Neil $\mathrm{BJ}$, et al. Clinical characteristics, management, and outcomes of patients diagnosed with acute pulmonary embolism in the emergency department: initial report of EMPEROR (Multicenter Emergency Medicine Pulmonary Embolism in the Real World Registry). J Am Coll Cardiol. 2011;57(6):700-6. https://doi.org/10.1016/j.jacc.2010.05.071

7. Volschan A, Albuquerque DC, Tura BR, Knibel Mde F, Souza PC Toscano ML. Pulmonary embolism: multicenter registry in tertiary hospitals. Rev Bras Ter Intensiva. 2009;21(3):237-46.

8. Volschan A, Albuquerque D, Tura BR, Knibel M, Esteves JP, Bodanese LC, et al. Predictors of hospital mortality in hemodynamically stable patients with pulmonary embolism. Arq Bras Cardiol. 2009;93(2):13540.

9. Laporte S, Mismetti P, Décousus H, Uresandi F, Otero R, Lobo JL, et al. Clinical predictors for fatal pulmonary embolism in 15,520 patients with venous thromboembolism: findings from the Registro
Informatizado de la Enfermedad TromboEmbolica venosa (RIETE Registry. Circulation. 2008;117(13):1711-6. https://doi.org/10.1161/ CIRCULATIONAHA.107.726232

10. Goldhaber SZ, Visani L, De Rosa M. Acute pulmonary embolism: clinical outcomes in the International Cooperative Pulmonary Embolism Registry (ICOPER). Lancet. 1999;353(9162):1386-9. https://doi.org/10.1016/S0140-6736(98)07534-5

11. Elias A, Mallett S, Daoud-Elias M, Poggi JN, Clarke M. Prognostic models in acute pulmonary embolism: a systematic review and metaanalysis. BMJ Open. 2016;6(4):e010324. https://doi.org/10.1136/ bmjopen-2015-010324

12. Erkens PM, Gandara E, Wells PS, Shen AY, Bose G, Le Gal G, et al. Does the Pulmonary Embolism Severity Index accurately identify low risk patients eligible for outpatient treatment? Thromb Res. 2012;129(6):710-4. https://doi.org/10.1016/j.thromres.2011.08.025

13. Fermann GJ, Erkens PM, Prins MH, Wells PS, Pap ÁF, Lensing AW Treatment of pulmonary embolism with rivaroxaban: outcomes by simplified Pulmonary Embolism Severity Index score from a post hoc analysis of the EINSTEIN PE study. Acad Emerg Med. 2015;22(3):299-307. https://doi.org/10.1111/acem.12615

14. Dubie E, Pouzet G, Bohyn E, Meunier C, Wuyts A, Chateigner Coelsch $\mathrm{S}$, et al. Outpatient management of pulmonary embolism diagnosed in emergency services [Article in French]. Ann Cardiol Angeiol (Paris) 2016;65(5):322-325. https://doi.org/10.1016/j.ancard.2016.09.013

15. Jiménez $D$, Yusen RD, Otero $R$, Uresandi $F$, Nauffal $D$, Laserna $E$ et al. Prognostic models for selecting patients with acute pulmonary embolism for initial outpatient therapy. Chest. 2007;132(1):24-30. https://doi.org/10.1378/chest.06-2921

16. Jiménez D, Aujesky D, Moores L, Gómez V, Lobo JL, Uresandi F, et al. Simplification of the pulmonary embolism severity index for prognostication in patients with acute symptomatic pulmonary embolism. Arch Intern Med. 2010;170(15):1383-9. https://doi. org/10.1001/archinternmed.2010.199

17. Venetz $C$, Jiménez $D$, Mean $M$, Aujesky $D$. A comparison of the original and simplified Pulmonary Embolism Severity Index. Thromb Haemost. 2011;106(3):423-8. https://doi.org/10.1160/TH11-04-0263 